\title{
Methods of Differential Submarine Detection Based on Magnetic Anomaly and Technology of Probes Arrangement
}

\author{
Yuqin Chen ${ }^{1,2, a}$, Jiansheng Yuan ${ }^{3, b}$ \\ ${ }^{1}$ State Grid Jiangsu Electric Power Research Institute, Nanjing 210019, China \\ ${ }^{2}$ State Grid Key Laboratory of Electric Power Metrology, Nanjing 210019, China \\ ${ }^{3}$ Department of Electrical Engineering, Tsinghua Univercity, Beijing 100084, China \\ achen_yq09@126.com, byuan@tsinghua.edu.cn
}

Keywords: Submarine detection, Differential detection, Multi-probes system

\begin{abstract}
Magnetic anomaly submarine detection methods detect submarines by the additional magnetic field generated by the ferromagnetic shell. Using a single magnetic measurement probe to detect submarines has difficult to overcome the background signal interference of geomagnetic field. So, this paper propose multi-probe method based on differential detection principle. It's advantage is that it can filter out the background noise, but as the magnetic field is directional, this approach has problem of blind zone. To overcome the problem of blind zone, this paper proposes two solutions. By comparing the submarine detection range of the two schemes, the detection system with four probes in two detection directions is demonstrated to have better detection effect, which overcomes the problem of blind zone and makes the detection distance farther.
\end{abstract}

\section{Introduction}

Magnetic anomaly submarine detection methods detect submarines by the additional magnetic field generated by the ferromagnetic shell. Currently many shipborne and airborne magnetic probes are used to search submarine actively by several countries ${ }^{[1]}$. By analyzing the magnetic signal from the probe in motion, the submarine is detected and positioned ${ }^{[2-3]}$.In this paper, the magnetic field in a region is monitored by arranging the probe network. By methods of differential detection and technology of probes arrangement, the problem of geomagnetic interference is solved and detection range is optimized.

\section{Magnetic Field Analysis}

To detect the submarine, the characteristics of the submarine induced magnetic field and marine background magnetic field should be analyzed.

Marine Magnetic Field. The marine magnetic field consists of two parts: the geomagnetic field and the induced magnetic field. The geomagnetic field contains the basic component which is stable $(40-60 \mu \mathrm{T})$, and the changing component(1-100nT) changes from time to time. The induced magnetic field(20-30nT) is caused by the tide ,sea wave ,etc.

Submarine Induced Magnetic Field. The magnetic field generated by the submarine can be divided into two categories: the intrinsic magnetic component and the induced magnetic component. As a result of long-term accumulation, intrinsic magnetic component can be degaussed in degaussing station. The induced magnetic component is induced by the geomagnetic field from time to time, it can be estimated by submarine size and magnetic field. For example, the magnetic moment of a submarine(100m in length, $10 \mathrm{~m}$ in width) is about $10^{7} \mathrm{~A}^{2} \mathrm{~m}$, the induced magnetic flux density at different distances on the long axis is shown in Table 1. If the background magnetic interference can't be eliminated, the detection distance is less than $500 \mathrm{~m}$.

Tab 1 Induced magnetic flux density of submarine with distance

\begin{tabular}{cccccc}
\hline Distance $(\mathrm{km})$ & 0.5 & 1 & 2 & 3 & 5 \\
\hline magnetic flux density $(\mathrm{nT})$ & 13.33 & 1.65 & 0.21 & 0.07 & 0.01 \\
\hline
\end{tabular}




\section{Differential Detection Method}

Because of the large variation of the background magnetic field, it is difficult to overcome the background magnetic field when using a single probe to detect the submarine. To overcome the problem of background magnetic interference, differential detection method is used.

Detection Principle. Although the magnetic field changes with the time, the magnetic field changes synchronously in a region. Therefore, the background magnetic interference can be eliminated by differing the signals of probe at different position. If there're two probes, the result should be $\left|B_{\mathrm{t} 1}-B_{\mathrm{t} 2}\right|$. If there're more probes, the result is shown in formula (1). Before detection, a threshold should be set, when the measured results exceed the threshold, it is believed that there is a submarine.

$$
\Delta B_{t}=\sqrt{\frac{\sum_{i \neq j}\left(B_{t i}-B_{t j}\right)^{2}}{n-1}}
$$

Measurement Selection. Magnetic field is vector field, we can either measure the total value, or the value in a certain direction. As the magnetic field signal is superimposed on the background magnetic field signal, and the magnetic field is very large, so that if the total value is measured, the submarine signal in the direction of the background magnetic field will be eliminated by differential method. Therefore in this paper, the submarine will be detected by measuring the magnetic field in certain directions.

\section{Probes Arrangement Technology}

The simplest differential detection system is composed of two magnetic field measurement probe, which has the problem of blind zone. Therefore, more probes and reasonable arrangement are needed.

Probes measuring $\boldsymbol{x}$ component. In order to analyze the detection effect of the multi-probe system, the detection performance of the single probe is analyzed firstly. The submarine is represented by a magnetic dipole point to $x$ axis, the probe measuring $\mathrm{x}$ component is set at $o(0,0)$ and the threshold is set as $1 \mathrm{nT}$.

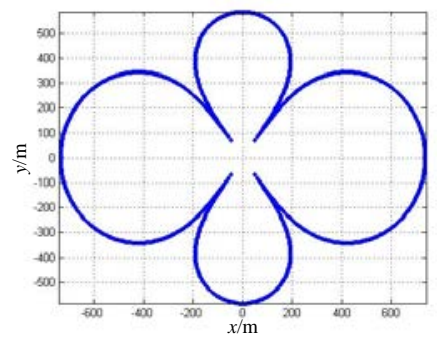

Fig.1 The detection range of a single probe

Fig. 1 shows the detection range of a single probe, which is calculated by comparing the signal generated by the submarine and the threshold value. It's easy to see that there're blind zones of detection in some direction.

In the actual detection, at least two probes are used to eliminate background interference, Fig.2 shows the detection range of two probes, the two probes are set at $( \pm 500,0)$. Blind zones still exist.

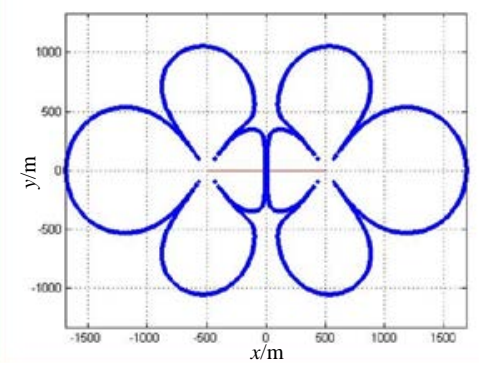

Fig. 2 The detection range of two probes 
In order to solve the problem of detecting blind zones, more probes should be used. Fig.3 shows the detection range of three-probe system and four-probe system. The probes are set at the vertex of the polygons in red, the solid line represents the detection range of four-probe system and the dotted line represents the detection range of three-probe system. The problem of detecting blind zones is solved by the increase of the probes, but the detection range is not large.

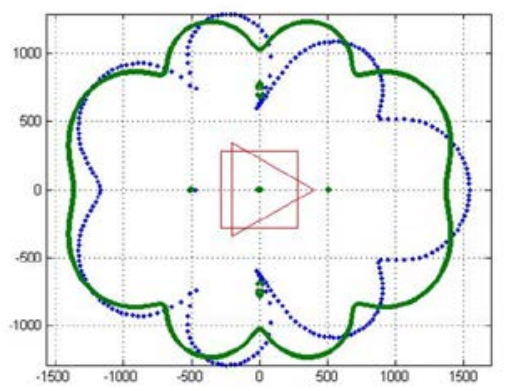

Fig.3 The detection range comparison between three-probe system and four-probe system

Probes measuring $x \boldsymbol{\&} y$ component. The detection range of probes measuring the $y$ component is different from probes measuring the $x$ component. Fig. 4 shows the detection range of a single probe in diffident probing direction, which are interlaced. If probes measuring both $x$ and $y$ components, there won't be any blind zones.

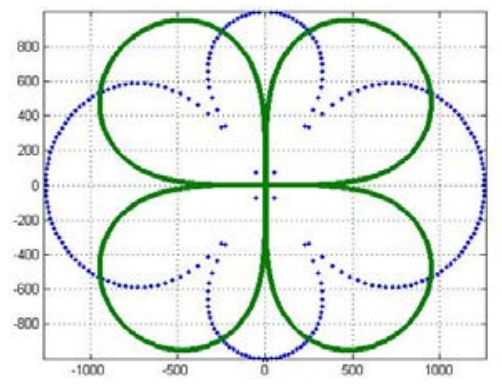

Fig.4 The comparison of detection range of single probe in diffident probing direction

Based on differential method, at least four probes should be used to measuring the both components, two for $x$ direction and two for $y$ direction. Fig. 5 shows the detection range of detection system within two-direction and two-points, the four probes are set at the end points of the red line. It's easy to see that the edge of the detection range has been improved. In order to increase the detection range, the distance between the two measuring points can be increased.

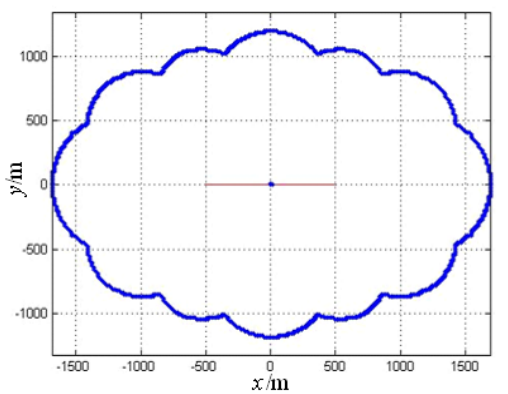

Fig.5 The detection range of detection system within two-direction and two-points

As the threshold determines the detection distance, Tab.2 shows the detection distance with threshold of two-direction and two-points detection system. Currently, the sensitivity of fluxgate probe is of order $10 \mathrm{pT}^{[4]}$, the detection distance may reach the order of $10 \mathrm{~km}$.

Tab.2 Detection distance with threshold

\begin{tabular}{cccccc}
\hline Threshold(nT) & 5 & 1 & 0.5 & 0.1 & 0.01 \\
\hline detection distance $(\mathrm{km})$ & 1100 & 2200 & 3000 & 5000 & 11000 \\
\hline
\end{tabular}




\section{Summary}

Single probe system has the problem of background noise, which can be solved by multi-probe system based on differential method. There're blind zones of detection when only two probes are used. More probes measuring one direction and probes measuring two different direction can both solve the problem of blind zone, and the latter one has a greater detection range.

\section{References}

[1]. Cui Guo-heng,Yu De-xin, Status quo of Non-acoustics Antisubmarine Detecting Technology and Its Countermeasures. Fire Control and Command Control, 2007, 12: 004.

[2]. Ginzburg, Boris, L. Frumkis, and B. Z. Kaplan. Processing of magnetic scalar gradiometer signals using orthonormalized functions. Sensors \& Actuators A Physical102(2002):67-75.

[3]. Sheinker, A., et al. Magnetic Anomaly Detection Using High-Order Crossing Method. Geoscience \& Remote Sensing IEEE Transactions on50.4(2012):1095-1103.

[4]. Edelstein A. Advances in magnetometry. Journal of Physics: Condensed Matter, 2007, 19(16): 165-217. 